\title{
Separation of Different Contributions to the Total X-ray Luminosity in Gamma-ray Loud Blazars
}

\author{
Jun-Hui Fan ${ }^{1,2}$, Gustavo E. Romero ${ }^{3}$, Yong-Xiang Wang ${ }^{4}$ and Jiang-Shui Zhang ${ }^{1}$ \\ 1 Center for Astrophysics, Guangzhou University, Guangzhou 510400; fjh@gzhu.edu.cn \\ 2 National Astronomical Observatories, Chinese Academy of Sciences, Beijing 100012 \\ 3 Instituto Argentino de Radioastronomía, C.C.5, (1894) Villa Elisa, Bs. As., Argentina \\ ${ }^{4}$ College of Science and Trade, Guangzhou University, Guangzhou 511442
}

Received 2005 January 6; accepted 2005 June 6

\begin{abstract}
The relativistic beaming model has been successfully used to explain many of the observational properties of active galactic nuclei. In this model the total emission is formed by two components, one beamed, one unbeamed. However, the exact contribution from each component in unresolved sources is still not clear. In the radio band, the core and extended emissions are clearly separated. We adopt the method proposed by Kembhavi to separate the two contributions in the X-ray emissions in a sample of 19 gamma-ray loud blazars. It is clearly shown that the beamed emission dominates the X-ray flux and the unbeamed X-ray emission is correlated with the extended radio emission of the considered objects. We also find that the ratio of the beamed to the unbeamed X-ray luminosity is correlated with the X-ray spectral index, an effect that should be a consequence of the underlying $\mathrm{X}$-ray emission mechanism.
\end{abstract}

Key words: galaxies: active-galaxies - jets $-\mathrm{X}$-rays: galaxies

\section{INTRODUCTION}

Urry \& Shafer (1984) proposed that the total emission from AGNs can be separated into two components, namely a beamed and an unbeamed component. Then, the observed total luminosity, $L^{\text {tot }}$, is the sum of the unbeamed, $L_{\mathrm{unb}}$, and beamed, $L_{\mathrm{b}}$, emissions, i.e., $L^{\text {tot }}=$ $L_{\text {unb }}+L_{\mathrm{b}}$.

In the radio region, the two contributions can be separated by VLBI observations, and the ratio of the beamed to unbeamed (or extended, $L_{\text {ext }}$ ) luminosities defines the core-dominance parameter $R=\frac{L_{\mathrm{b}}}{L_{\mathrm{unb}}}$ (or $\frac{L_{\mathrm{b}}}{L_{\mathrm{ext}}}$ ). In the X-ray band, the observed emission should also be separable into two parts as was assumed by Brown \& Murphy (1987), but the lack of angular resolution in the observations of most sources renders a clear separation not easy. However, some methods have been recently proposed to achieve an adequate separation of the different contributions.

In the context of the radio beaming model it was assumed that the ratio of the beamed radio emission, in the transverse direction, to the extended radio emission is a constant (Orr \& Brown 
1982). Browne \& Murphy (1987) applied this idea to the X-ray luminosity and assumed that the transverse beamed X-ray luminosity $\left(L_{\mathrm{x}, \mathrm{b}}(90 \mathrm{deg})\right)$ is also proportional to the extended radio emission $\left(L_{\mathrm{r}, \mathrm{unb}}\right)$, i.e., $L_{\mathrm{x}, \mathrm{b}}(90 \mathrm{deg})=A L_{\mathrm{r} \text {, unb }}$, with $A=$ constant. The beamed luminosity for an inclination angle $\psi$ between the beam direction and the line of sight is $L_{\mathrm{x}, \mathrm{b}}(\psi)=$ $g_{\mathrm{x}}(\beta, \psi) L_{\mathrm{x}, \mathrm{b}}(90 \mathrm{deg})$, where $g_{\mathrm{x}}(\beta, \psi)$ is the $\mathrm{X}$-ray beaming factor, given by

$$
g_{x}(\beta, \psi)=\frac{1}{2}\left[(1-\beta \cos \psi)^{-\left(2+\alpha_{\mathrm{x}}\right)}+(1+\beta \cos \psi)^{-\left(2+\alpha_{\mathrm{x}}\right)}\right] .
$$

In this expression, $\beta$ is the relativistic bulk velocity in units of $c$ and $\alpha_{\mathrm{x}}$ is the spectral index of the beamed X-ray emission. Here, $\beta \cos \psi$ can be obtained for each source from the following relation

$$
R_{\text {radio }}=R_{90 \operatorname{deg}} \frac{1}{2}\left[(1-\beta \cos \psi)^{-2}+(1+\beta \cos \psi)^{-2}\right],
$$

which is valid for flat-spectrum radio AGNs. $R_{\text {radio }}=\frac{L_{\mathrm{r}, \mathrm{b}}}{\mathrm{Lr} \text { ext }}$ is a core-dominance ratio and $R_{90} \mathrm{deg}$ was found to be 0.024 by Orr \& Browne (1982) from numerical fits of their sample. The ratio of the beamed to unbeamed X-ray luminosity is

$$
R_{\mathrm{x}}=\frac{L_{\mathrm{x}, \mathrm{b}}}{L_{\mathrm{x}, \mathrm{unb}}}=R_{\mathrm{tx}} g_{\mathrm{x}}(\beta, \psi), R_{\mathrm{tx}}=\frac{L_{\mathrm{x}, \mathrm{b}}(90 \mathrm{deg})}{L_{\mathrm{x}, \mathrm{b}}} .
$$

Here, $R_{\mathrm{tx}}$ is assumed to be a constant.

In 1987, Browne and Murphy proposed a model, in which they assumed that the X-ray emission is proportional to the extended radio emission and the correlation between the X-ray and extended radio emissions is from the observed X-ray luminosity and radio luminosity(see Kembhavi \& Narlikar 1999). Later on, Kembhavi (1993) proposed a beaming model that uses the formalism suggested by Browne and Murphy but with a different scheme for separating the X-ray luminosity into beamed and isotropic parts. Kembhavi (1993) chose a subset of 34 quasars with $\frac{L_{\mathrm{r}, \mathrm{b}}}{L_{\mathrm{r}, \text { unb }}}>10$ from Browne and Murphy's sample, and found a significant correlation between $\log L_{\mathrm{x}}$ and $\log L_{\mathrm{r}, \mathrm{b}}$. Because the fit is dominated by beamed emission, the $\log L_{\mathrm{x}}-$ $\log L_{\mathrm{r}, \mathrm{b}}$ relation suggests that there may also be a relation between the beamed X-ray and radio components. Since the launch of Gamma Ray Observatory (GRO) in the early 1990s, many active galactic nuclei (AGNs) have been detected at the $E>100 \mathrm{MeV}$ band with the EGRET instrument (Hartman et al. 1999). Gamma-ray emission is one of the most interesting topics in AGN research, but the exact nature of the emission mechanism is still an open problem. Rapid variability observed in some sources and $\gamma-\gamma$ opacity arguments suggest that the gamma-ray emission is highly beamed. In the present paper, we will apply Kembhavi's proposal to a sample of 19 gamma-ray loud blazars with $\frac{L_{\mathrm{r}, \mathrm{b}}}{L_{\mathrm{r}, \mathrm{unb}}}>10$ in order to establish the beaming level of X-ray emission for these sources.

\section{SAMPLE}

From the available literature, we selected a sample of $\gamma$-ray loud blazars with $\frac{L_{\mathrm{r}, \mathrm{b}}}{L_{\mathrm{r}, \mathrm{unb}}}>10$. The relevant information (along with the results described in the next section) is listed in Table 1. In this table column 1 gives the object name; column 2, the redshift $(z)$; column 3 , the core luminosity at $5 \mathrm{GHz}$ in units of $\mathrm{W} \mathrm{Hz}^{-1}$; column 4 , the extended luminosity at $5 \mathrm{GHz}$ in units of $\mathrm{W} \mathrm{Hz}^{-1}$; column 5 , the references for the radio data; column 6 , the X-ray luminosity at $1 \mathrm{keV}$ in units of $\mathrm{W} \mathrm{Hz}^{-1}$; column 7 , the corresponding references for the X-ray data; column 8 , the X-ray spectral index; and finally column 9 , the ratio of the beamed to the unbeamed X-ray luminosities $R_{\mathrm{x}}=\frac{L_{\mathrm{x}, \mathrm{b}}}{L_{\mathrm{x}, \mathrm{unb}}}$. 
Table 1 A Sample of $19 \gamma$-ray Loud Blazars

\begin{tabular}{|c|c|c|c|c|c|c|c|c|}
\hline $\begin{array}{l}\text { Name } \\
\text { (1) }\end{array}$ & $\begin{array}{c}\mathrm{Z} \\
(2)\end{array}$ & $\begin{array}{l}L_{\mathrm{r}, \mathrm{b}}{ }^{a} \\
\quad(3)\end{array}$ & $\begin{array}{l}L_{\mathrm{r}, \text { unb }} \\
\quad(4)\end{array}$ & $\begin{array}{l}\text { Ref } \\
(5)\end{array}$ & $\begin{array}{c}L_{\mathrm{x}}^{\text {tot } a} \\
(6)\end{array}$ & $\begin{array}{l}\text { Ref } \\
(7)\end{array}$ & $\begin{array}{l}\alpha_{\mathrm{x}} \\
(8)\end{array}$ & $\begin{array}{l}R_{\mathrm{x}} \\
(9)\end{array}$ \\
\hline $0234+285$ & 1.207 & 28.20 & 26.20 & $\overline{\mathrm{BM}}$ & 21.050 & C97 & 0.67 & 499.53 \\
\hline $0235+164$ & 0.940 & 27.61 & 26.41 & $\mathrm{Mu}$ & 21.971 & C97 & 1.57 & 1029.74 \\
\hline 0336-019 & 0.852 & 28.00 & 26.50 & $\mathrm{BM}$ & 20.710 & C97 & 0.67 & 116.10 \\
\hline 0420-014 & 0.915 & 28.20 & 25.80 & $\mathrm{BM}$ & 21.430 & C97 & 0.67 & 1669.84 \\
\hline $0537-441$ & 0.894 & 28.20 & 25.90 & $\mathrm{BM}$ & 21.810 & C97 & 1.16 & 12454.35 \\
\hline $0735+178$ & 0.424 & 27.18 & 25.37 & $\mathrm{Mu}$ & 20.474 & C97 & 1.25 & 3120.11 \\
\hline $0804+499$ & 1.430 & 27.84 & 26.24 & $\mathrm{Mu}$ & 21.474 & C97 & 0.56 & 99.80 \\
\hline $0851+202$ & 0.306 & 26.81 & 24.03 & K92 & 20.768 & C97 & 1.45 & 552049.89 \\
\hline $1127-145$ & 1.187 & 28.50 & 26.80 & $\mathrm{BM}$ & 21.610 & C97 & 0.67 & 218.23 \\
\hline $1222+216$ & 0.435 & 26.48 & 25.48 & H92 & 20.538 & C97 & 0.67 & 22.72 \\
\hline $1253-055$ & 0.538 & 28.10 & 27.00 & $\mathrm{BM}$ & 21.400 & C97 & 0.83 & 54.77 \\
\hline 1510-089 & 0.361 & 27.30 & 25.80 & $\mathrm{BM}$ & 20.750 & C97 & 0.90 & 288.87 \\
\hline $1611+343$ & 1.401 & 28.40 & 27.00 & $\mathrm{BM}$ & 21.680 & C97 & 0.76 & 110.66 \\
\hline $1633+382$ & 1.814 & 28.60 & 26.70 & $\mathrm{BM}$ & 22.120 & C97 & 0.53 & 213.19 \\
\hline $1730-130$ & 0.908 & 28.30 & 26.50 & $\mathrm{BM}$ & 21.580 & C97 & 0.67 & 262.38 \\
\hline $1739+522$ & 1.375 & 27.90 & 26.20 & $\mathrm{BM}$ & 21.450 & C97 & 0.67 & 218.23 \\
\hline $2032+107$ & 0.601 & 27.11 & 26.02 & $\mathrm{AU}$ & 20.216 & C97 & 1.43 & 440.27 \\
\hline $2230+114$ & 1.037 & 28.30 & 26.90 & $\mathrm{BM}$ & 21.390 & C97 & 0.67 & 78.49 \\
\hline $2251+158$ & 0.559 & 28.60 & 27.40 & $\mathrm{BM}$ & 21.410 & C97 & 0.62 & 34.48 \\
\hline
\end{tabular}

Refs. to Table 1:

a: in units of $\mathrm{W} \mathrm{Hz}^{-1}$; AU: Antonucci \& Ulvestad (1985); BM: Browne \& Murphy (1987); C97: Comastri et al. (1997); Mu: Murphy et al. (1993); K92: Kollgaard et al. (1992); H92: Hooimeyer et al. (1992).

\section{RESULTS}

Following Kembhavi's proposal, for the relevant data listed in Table 1, we can obtain a linear regression correlation

$$
\log L_{\mathrm{x}}=(0.64 \pm 0.14) \log L_{\mathrm{r}, \mathrm{b}}+3.49 .
$$

The total X-ray luminosity should be formed by two components. We assume that the linear correlation holds for all gamma-ray loud blazars, and can be expressed as

$$
\log L_{\mathrm{x}}=0.64 \log L_{\mathrm{r}, \mathrm{b}}+\log k
$$

where $k$ is a constant. In this sense, the total X-ray luminosity is given by

$$
\begin{gathered}
L_{\mathrm{x}}^{\mathrm{tot}}=L_{\mathrm{x}, \mathrm{b}}+L_{\mathrm{x}, \mathrm{unb}}=L_{\mathrm{x}, \mathrm{b}}\left(1+\frac{1}{R_{\mathrm{tx}} g_{\mathrm{x}}(\beta, \psi)}\right) \\
=k L_{\mathrm{r}, \mathrm{b}}^{0.64}\left(1+\frac{1}{R_{\mathrm{tx}} g_{\mathrm{x}}(\beta, \psi)}\right),
\end{gathered}
$$

where $L_{\mathrm{x}, \mathrm{unb}}=L_{\mathrm{x}, \mathrm{b}} / R_{\mathrm{tx}} g_{\mathrm{x}}(\beta, \phi)$.

The two constants, $\log k=3.48$, and $R_{\mathrm{tx}}=\frac{L_{\mathrm{x}, \mathrm{b}}(90 \mathrm{deg})}{L_{\mathrm{x}, \mathrm{u}}}=5.9 \times 10^{-3}$ are determined by minimizing $\Sigma\left[\log \left(L_{\mathrm{x}} / L_{\mathrm{x}}^{\text {tot }}\right)\right]^{2}$ for the objects in our sample as Browne \& Murphy (1987) and Kembhavi (1993) did. The ratio of the two components in X-ray band, $R_{\mathrm{x}}$, which can be obtained from relations (1) and (3), is listed in column 9 of Table 1. 


\section{DISCUSSION}

Blazars are characterized by large and violent flux variations, high and variable polarization, predominantly non-thermal emission over almost the entire electromagnetic spectrum and relativistic electrons tangled with magnetic field in a relativistic jet nearly pointing towards us. The blazar phenomenon can be described with a jet plus a supermassive black hole surrounded with an accretion disk (see Urry \& Padovani 1995; Wang 2003). The EGRET on Gamma-Ray Observatory (GRO) has detected about 120 possible blazars (Yang \& Fan 2005). Many authors have investigated the gamma-ray emission mechanism. They showed that both the synchrotron self-Compton (SSC) and the external inverse Compton (EIC) processes are good possibilities to explain the gamma-ray emission, but it is not determined yet which one is the case in most gamma-ray sources. If the beamed and the unbeamed emission in X-rays follow those in radio bands, then it will favor the SSC model since the gamma-ray emission is strongly beamed. In the present paper, we chose the gamma-ray loud blazars as our sample in order to separate the beamed and the unbeamed X-ray emissions from the observed data.

According to the standard model, the emission of blazars at different wavebands should be from two components, something that was used by several authors to explain the observational properties of blazars. Based on the model, Fan (2003) proposed a method to calculate the intrinsic ratio of the luminosity in the jet to the unbeamed luminosity in the co-moving frame, and found that the average ratio in BL Lacertae objects is greater than that in flat spectrum radio quasars. Different average ratios probably give rise to different observed emission line strengths in BL Lacertae objects, which show no emission lines or very weak emission lines and in flat spectrum radio quasars that show very strong emission lines. When we considered the effect of the ratio $f$ in the polarization-core-dominance parameter plot, the BLs and FSRQs satisfy a similar correlation, which suggests that the polarization nature is quite similar in BLs and FSRQs (Fan et al. 2004). At the X-ray band, the emission should also be formed by two components, but they are hard to separate. Kembhavi (1993) found a tight correlation between radio core-luminosity and the X-ray luminosity, giving a key to separate the X-ray different types of emission. It is then possible to discuss the relationship between X-ray and radio emission of the core and the extended regions.

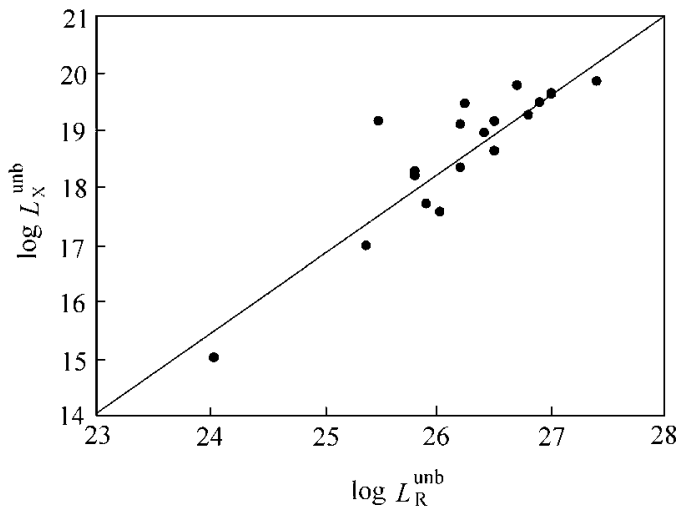

Fig. 1 Plot of unbeamed X-ray luminosity ( $\mathrm{W} \mathrm{Hz}^{-1}$ ) against unbeamed radio luminosity $\left(\mathrm{W} \mathrm{Hz}^{-1}\right)$. The solid line represents the best fitting result.

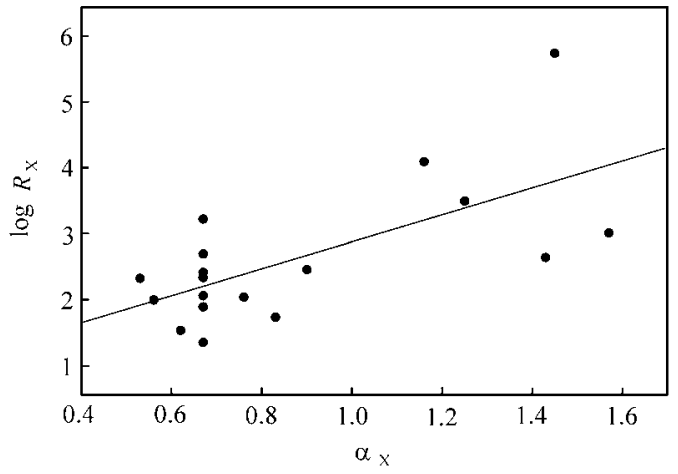

Fig. 2 Plot of ratio $\mathrm{R}_{X}$ against $\mathrm{X}$-ray spectral index $\alpha_{X}$. The solid line represents the bet fitting result. 


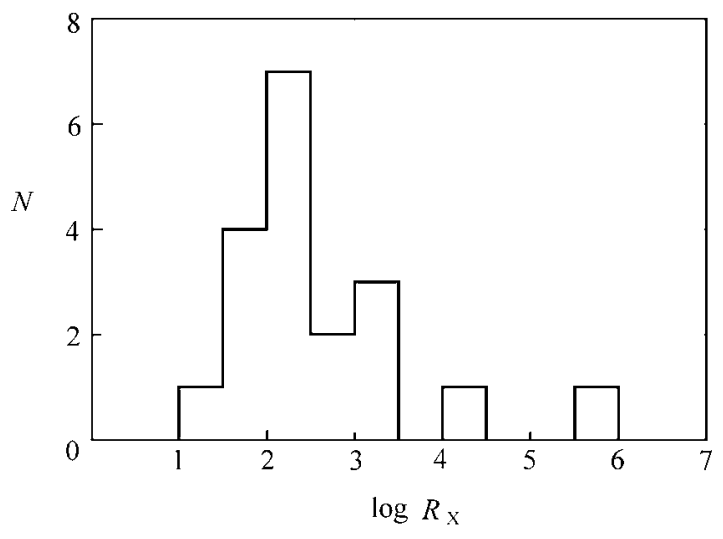

Fig. 3 Histogram of ratio $R_{\mathrm{X}}$.

In Kemhavi's work, $R_{\mathrm{xt}}$ was found to be $1.3 \times 10^{-2}$, which is twice our value of $5.9 \times 10^{-3}$. The difference is not large which is understandable since our and his samples are both of radio loud sources. Our $R_{x t}$ gives the ratio $\mathrm{R}_{\mathrm{x}}$ as listed in column 9 of Table 1 , from which one can obtain the extended (unbeamed) X-ray emission, $\log L_{\mathrm{x}}$ unb $=\log L_{\mathrm{x}}^{\text {tot }}-\log R_{\mathrm{x}}$. The extended (unbeamed) X-ray luminosity is found to be correlated with the extended radio luminosity as shown in Figure $1, \log L_{\mathrm{x}}$, unb $=(1.39 \pm 0.18) \log L_{\mathrm{r} \text {, unb }}-(17.92 \pm 4.6)$ with a correlation coefficient $r=0.787$ and a chance probability $p=4.22 \times 10^{-7}$. This correlation may come from the fact that $\log L_{\mathrm{x}}$, unb and $\log L_{\mathrm{r} \text {, unb }}$ are both closely associated with the redshift. This can be tested if we use Padovani's method (Padovani 1992, see also Fan et al. 1996) to deal with the relevant data. If $r_{i j}$ is the correlation coefficient between $\mathrm{x}_{i}$ and $\mathrm{x}_{j}$, in the case of three variables the correlation between two of them, excluding the effect of the third one, is

$$
r_{12,3}=\frac{r_{12}-r_{13} r_{23}}{\sqrt{\left(1-r_{13}^{2}\right)\left(1-r_{23}^{2}\right)}} .
$$

When the relevant data listed in Table 1 are used to the above formulae, one can find that

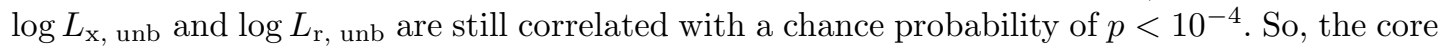
$\mathrm{X}$-ray and radio emissions are correlated and the extended ones are also correlated. It perhaps favors the SSC model for the gamma-ray emission.

In the X-ray region, the spectral index is found to change from one source to the next. Worrall \& Marshall (1984) found that BLs show steeper X-ray spectral index than flat spectrum radio sources. Later, Comastri et al. (1997) confirmed that result. It is possibly caused by the emission mechanism of the X-rays. In BL Lacertae objects, the X-ray emission is probably from the synchrotron tail and shows a steeper spectrum, whereas in flat spectrum radio quasars the X-ray emission is thought to originate from inverse Compton scattering and even, to some extent, from thermal processes in the innermost region, so it shows a flatter spectrum. If this is true, one can expect that the X-rays in BL Lacertae objects are mainly from the jet, hence displaying a high ratio $\mathrm{R}_{X}$, while for flat spectrum radio quasars, the $\mathrm{X}$-rays would be from both the jet and the accretion disk (see Grandi \& Palumbo 2004), giving a lower ratio $R_{\mathrm{x}}$. Therefore, a correlation between $R_{\mathrm{x}}$ and $\alpha_{\mathrm{x}}$ can be expected. This is shown in Figure 2, using the data of Table 1. The solid line represents the best fit result, $\log R_{\mathrm{x}}=(2.04 \pm 0.55) \alpha_{\mathrm{x}}+0.83 \pm 0.51$ with a correlation coefficient $r=0.443$ and a chance probability $p=2 \times 10^{-3}$. Although the points are somehow scattered, there seems to be a real trend for steeper X-ray spectra to correspond to higher $R_{\mathrm{x}}$ ratio, as expected. In flat spectrum radio quasars, if the $\mathrm{X}$-ray emission consists of 
an inverse Compton contribution plus other emissions of different origin, then it is more difficult to expect that these objects could present inverse Compton $\mathrm{TeV}$ emission. In contrast, in $\mathrm{BL}$ Lacertae objects, the synchrotron emission reaches the X-ray range, then one can expect that the inverse Compton emission can reach the $\mathrm{TeV}$ domain. Observations indicate that the $\mathrm{TeV}$ gamma-ray blazars are all BL Lacertae objects being consistent with these expectations.

From Table 1, one can find that the average ratio $R_{\mathrm{x}}$ of the sources considered in the present paper satisfies $\log R_{\mathrm{x}}=2.60 \pm 1.01$. The corresponding histogram for $R_{\mathrm{x}}$ is shown in Figure 3 . For core-dominated blazars, the X-ray emission is dominated by jets. Therefore, it is possible to ignore the extended contribution in X-ray studies of these objects.

We have separated the beamed and unbeamed contributions to the total X-ray emission for a sample of $19 \gamma$-ray loud blazars. We have found that 1) The ratio of the beamed to the unbeamed luminosity is $\left.\log \left\langle R_{\mathrm{x}}\right\rangle=2.60 \pm 1.01 ; 2\right)$ The unbeamed X-ray and radio emissions are correlated; and 3) The luminosity ratio is correlated with the X-ray spectral index.

Acknowledgements The authors thank the referee for his constructive suggestions and comments. This work was supported by the National 973 project (NKBRSF G19990754), the National Science Fund for Distinguished Young Scholars (10125313), and the Fund for Top Scholars of Guangdong Province (Q02114). J. H. Fan also thanks the support from the Guangzhou Education Committee. The GER research on high energy astrophysics is mainly supported by the Argentine agencies CONICET and ANPCT (PICT No. 03-13291).

\section{References}

Antonucci R. R. J., Ulvestad J. S., 1985, ApJ, 294, 158

Browne I. W. A., Murphy D. W., 1987, MNRAS, 226, 601

Comastri A. et al., 1997, ApJ, 480, 534

Fan J. H., 2003, ApJ, 585, L23

Fan J. H., Wang Y. J., Yang J. H., Su C. Y., 2004, ChJAA, 4, 533

Grandi P., Palumbo G. G. C., 2004, Sci, 306, 998

Kembhavi A., 1993, MNRAS, 264, 683

Kollgaard R. I. Wardle J. F. C., Roberts D. H. et al., 1992, AJ, 104, 1687

Murphy D. W., Browne I. W. A., Perley R. A, 1993, MNRAS, 264, 298

Hooimeyer J. R. A., Schilizzi R. T., Miley G. K. et al., 1992, A\&A, 261, 25

Orr M. J. L., Brown I. W. A., 1982, MNRAS, 200, 1067

Urry C. M., Shafer R. A., 1984, ApJ, 280, 569

Urry C. M., Padovani P., 1995, PASP, 107, 803

Valtaoja E., Lahteenmaki A., Terasranta H., Lainela, M., 1999, ApJS, 120, 95

Vermeulen R. C., Cohen M. H., 1994, ApJ, 430, 467

Veron-Cetty M.-P., Veron P., 1998, ESO Scientific Report, No. 18

Wang J.-M., 2003, AJ, 125, 2859

Wills B. J., Wills D., Breger M., Antonucci R. R. J., Barvainis R., 1992, ApJ, 398, 454

Wills B. J., Browne I. W. A., 1986, ApJ, 302, 56

Worrall D. M., Marshall F. E., 1984, ApJ, 276, 434

Yang J. H., Fan J. H., 2005, ChJAA, 5(3), 229

Zensus J. A., Pearson T. J., 1988, IAU Symp. 129, 7 